\title{
El papel de la adamalisina-17 (ADAM17) en la periodontitis
}

\author{
The role of adammalysin-17 (ADAM17) in periodontitis \\ David Gómez-Thomas, ${ }^{*}$ Ruth Rodríguez-Montaño, ${ }^{\ddagger}$ Sergio José Zepeda-Nuño, $\$$ \\ Alondra del Carmen Ruíz-Gutiérrez, ${ }^{*}$ Celia Guerrero-Velázquez ${ }^{\ddagger}$
}

\section{RESUMEN}

Las adamalisinas (ADAM) son proteínas con características de proteasas que regularmente se encuentran en la membrana de varios tipos celulares. Se ha descrito que las ADAM tienen la capacidad de escindir varias moléculas como receptores, citocinas y factores de crecimiento en su porción del ectodominio, convirtiendo varias moléculas en solubles que funcionan como activadoras 0 inhibidoras de diferentes procesos celulares. Se ha descrito que ADAM17 escinde de la membrana el factor de crecimiento tumoral $\alpha$ (TNF- $\alpha$ ), el ligando del receptor de NF-kB (RANKL) y el receptor de interleucina-23 (IL-23R) IL-23, convirtiéndolos en moléculas solubles que actúan de manera importante en la destrucción ósea. En este sentido se ha descrito que TNF- $\alpha$, RANKL y el IL-23R se encuentran aumentadas en varios tipos de muestras de pacientes con periodontitis. Esta revisión aborda el papel que juega la ADAM17 en la periodontitis, tomando en cuenta que esta molécula participa en la generación de la TNF- $\alpha$, RANKL y el IL-23R, moléculas involucradas en la destrucción de los tejidos de soporte en la periodontitis.

Palabras clave: Adamalisinas, ADAM17, periodontitis.

\section{ABSTRACT}

Adamalyins (ADAM) are proteins with characteristics of proteases, which are regularly found in the membrane of various cell types. ADAMs have been described as having the ability to cleave various molecules such as receptors, cytokines, and growth factors in their portion of the ectodomain, making various molecules soluble that function as activators or inhibitors of different cellular processes. ADAM17 has been described to cleave the membrane, tumor growth factor $\alpha$ (TNF- $\alpha$ ), the NF- $\kappa B$ receptor ligand (RANKL) and the interleukin-23 receptor (IL-23R) IL-23, converting them into soluble molecules, that act in an important way in bone destruction. In this sense, it has been described that TNF- $\alpha$, RANKL and IL$23 R$ are increased in various types of samples from patients with periodontitis. This review addresses the role that ADAM17 plays in periodontitis, taking into account that this molecule participates in the generation of TNF- $\alpha, R A N K L$ and IL-23R, molecules involved in the destruction of supporting tissues in periodontitis.

Keywords: Adamalysins, ADAM17, periodontitis.

\section{INTRODUCCIÓN}

Las enfermedades periodontales son un grupo de desórdenes con diferentes etiologías y manifestaciones clínicas. Dichas enfermedades incluyen la periodontitis, la cual se distingue por una fuerte respuesta inmunoinflamatoria caracterizada por la inflamación gingival, bolsas periodontales, pérdida de inserción y pérdida ósea radiográfica. ${ }^{1,2}$ En este contexto, la destrucción de los tejidos de soporte del diente es generada por varias células y moléculas del sistema inmune, como

* Especialidad de Periodoncia. Departamento de Clínicas Odontológicas Integrales.

‡ Instituto de Investigación en Odontología. Departamento de Clínicas Odontológicas Integrales.

$\S$ Laboratorio de Patología. Departamento de Microbiología y Patología.

Centro Universitario de Ciencias de la Salud. Universidad de Guadalajara. Guadalajara, Jalisco, México.

Recibido: 15 de diciembre de 2020. Aceptado: 16 de abril de 2021.

Citar como: Gómez-Thomas D, Rodríguez-Montaño R, Zepeda-Nuño SJ, Ruíz-Gutiérrez AC, Guerrero-Velázquez C. El papel de la adamalisina-17 (ADAM17) en la periodontitis. Rev Mex Periodontol. 2020; 11 (1-3): 10-15. https://dx.doi.org/10.35366/102641 
las citocinas proinflamatorias y sus receptores (IL-1, IL-6 y TNF- $\alpha$ ). También varios mecanismos están involucrados en la destrucción de los tejidos del periodonto como el eje IL-23/ IL-17 y el sistema RANK/RANKL/OPG. ${ }^{2}$

Recientemente, se ha descrito que las enzimas ADAM (del inglés a disintegrin and metalloproteinase) son una subfamilia de proteasas de las adamalisinas descritas inicialmente en 1995 por Wolfsberg y colaboradores. Estas adamalisinas están implicadas en un gran número de enfermedades, incluyendo la artritis reumatoide, la arterioesclerosis, asma y cáncer.

Las ADAM poseen aproximadamente de 750 a 800 aminoácidos de longitud, tienen actividad enzimática dependiente de zinc y sus funciones incluyen la adhesión celular y procesamiento proteolítico de la membrana extracelular, así como la escisión de ectodominios de diversos receptores celulares de superficie y moléculas de señalización. ${ }^{3}$ Se han identificado 38 miembros de la familia ADAM en diferentes especies. En el genoma humano existen 25 genes que codifican para ADAM, de los cuales cuatro son pseudogenes. De las 21 ADAM funcionales en los humanos, 13 poseen el sitio característico activo tipo reprolisina en el dominio de metaloproteinasa. ${ }^{4}$

\section{SÍNTESIS Y ESTRUCTURA DE LAS ADAM}

Después de la transcripción de los RNA mensajeros para las ADAM, éstos se translocan del núcleo hacia el retículo endoplásmico rugoso, y una vez traducidos los mensajeros en proteínas precursoras de las ADAM, son liberadas y transportadas al aparato de Golgi, en donde son glicosiladas y activadas a través de la remoción de su pro-dominio por parte de la proteína convertasa 7 o furina. ${ }^{5}$

Las ADAM poseen secuencias de señal en el extremo $\mathrm{N}$-terminal, que está conformado por cinco cadenas dispuestas en hoja beta plegada, dicho extremo está encargado de direccionar a las ADAM hacia la vía secretora. Este subdominio se encuentra seguido de un pro-dominio que actúa como una chaperona intramolecular que asegura el correcto plegado de la proteína, mediante el mecanismo de switch de cisteína mantiene a la enzima en un estado latente. Después del pro-dominio, las ADAM poseen un dominio catalítico altamente conservado de metaloproteinasas que consiste en una metzincina, la cual posee una estructura globular compuesta por dos subdominios. El átomo catalítico de zinc se encuentra en el fondo de la ranura entre los dos subdominios (Figura 1). ${ }^{3,6}$

El dominio de metaloproteinasa está seguido por un dominio tipo desintegrina (disintegrin-like), que participa en los procesos de adhesión celular. ${ }^{6,7}$ El dominio tipo desintegrina posee 14 aminoácidos y está implicado en las interacciones entre las ADAM y las integrinas. Cuando se identificó la primer ADAM en mamíferos se planteó que los dominios tipo desintegrina podrían interactuar con las integrinas, tal y como sucede con las secuencias de las metaloproteasas solubles del veneno de serpiente. ${ }^{3}$ Regularmente estas interacciones pueden influir en la adhesión celular, así como en la comunicación célula-célula. ${ }^{4}$

El extremo carboxilo de las ADAM se encuentra conformado por un dominio rico en cisteína que está involucrado en la regulación de su actividad catalítica, ${ }^{9}$ así como en el marcaje del sustrato. ${ }^{10}$ Seguido del dominio rico en cisteína se encuentra un dominio tipo factor de crecimiento epidérmico (EGF) ausente en ADAM10 y ADAM17. Después del dominio EGF le sigue una porción transmembranal y, por último, el tallo citoplasmático que en las ADAM puede variar enormemente en longitud y secuencia entre cada subtipo, desde 11 residuos en la ADAM11 hasta los 231 residuos en ADAM19. ${ }^{11}$ La porción transmembranal posee una o más secuencias PXXP que pueden servir como sitio de unión para proteínas que contienen dominios $\mathrm{SH} 3$ y residuos de serina, tirosina y treonina, $y$ a su vez son porciones que pueden ser fosforiladas por diversas cinasas. Esto lleva a especular que los dominios citoplasmáticos juegan un papel importante en la regulación de la función proteolítica en respuesta, no sólo a señales intracelulares, sino que, mediante la interacción de los ectodominios con sus sustratos, dan lugar al reclutamiento de moléculas de señal y adaptadoras. ${ }^{4}$

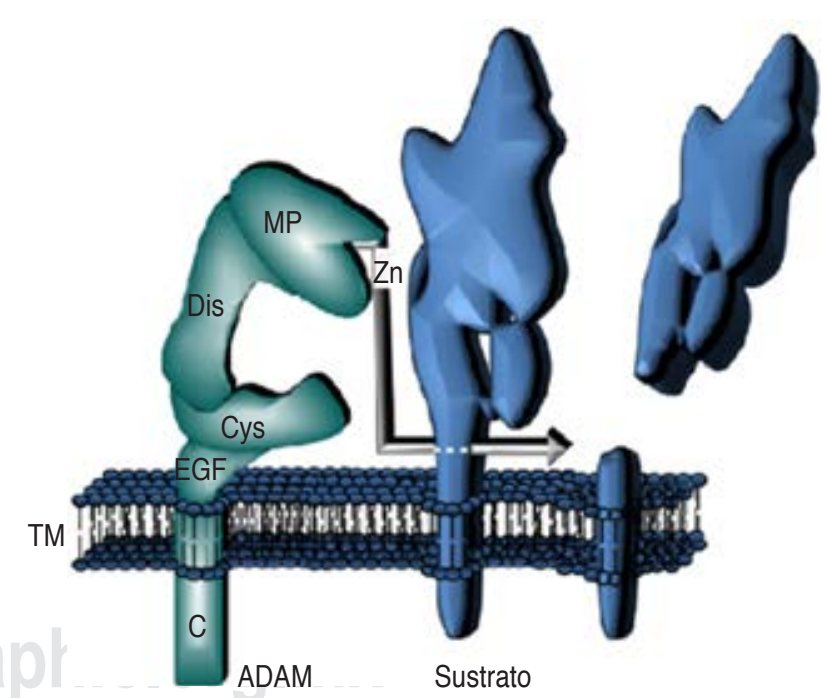

Figura 1: Actividad proteolítica de las ADAM. A través de su dominio de metaloproteasa ocasiona la escisión de múltiples proteínas de superficie, las cuales pueden tener efecto autocrino o paracrino. Modificada de: Saftig $P$ et al. ${ }^{8}$

MP = dominio metaloproteinasa; $\mathrm{Zn}$ = zinc; Dis = dominio desintegrina; Cys = dominio rico en cisteína; $\mathrm{EGF}=$ factor de crecimiento epidérmico; $\mathrm{TM}$ = transmembranal; $\mathrm{C}=$ porción citoplasmática; $\mathrm{ADAM}=$ del inglés a disintegrin and metalloproteinase. 
Figura 2:

Estructura y función de ADAM17.

La metaloproteasa ADAM17 puede estar dividida en seis dominios con distintas funciones, aquí separadas por colores diferentes. Modificada de: Zunke F et al. 2017. ${ }^{19}$ $\mathrm{ADAM}=$ del inglés a disintegrin and metalloproteinase; CANDIS $=$ del inglés conserved ADAM seventeen dynamic interaction sequence
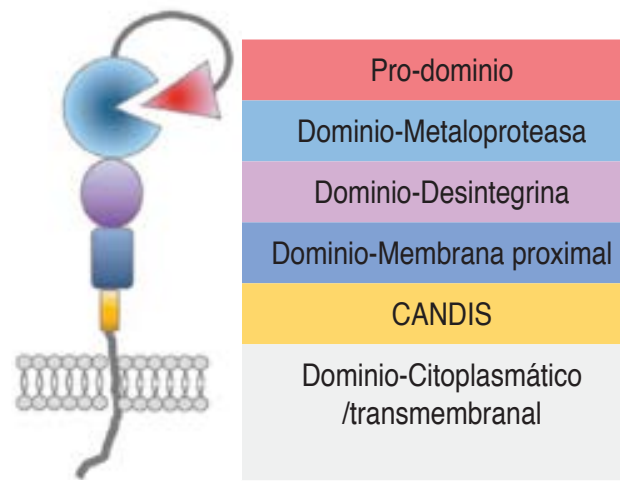

Inactivación y función de chaperona

Ectodominio de desprendimiento

Activación e interacción con integrinas

Posible reconocimiento de sustrato y activación

Posible reconocimiento de sustrato y activación

Localización transmembranal e intracelular, tráfico y activación mediante fosforilación e interacción con moléculas de señalización

\section{FUNCIONES DE LAS ADAM}

La familia de las ADAM posee una estructura compleja pero conservada. Están involucradas en varias funciones fisiológicas, tales como la adhesión, migración, proteólisis y señalización celular, regulando el fenotipo celular. Pueden modular las respuestas celulares mediante el procesamiento o escisión de diversos ectodominios (TNF- $\alpha$, EGF, ligando delta de Notch, etcétera). Además, participan en las interacciones célula-célula, la fertilización y formación de los tejidos nervioso, vascular, muscular y adiposo. ${ }^{12,13}$ Una alteración en la expresión de las ADAM puede jugar un papel importante y decisivo en diversos procesos patológicos como enfermedades cardiovasculares, neurodegenerativas, asma, infecciones, autoinmunes y cáncer. ${ }^{14}$

\section{ADAM17}

La ADAM17 fue descubierta por tener la función de escindir el TNF y se le denominó enzima convertidora del factor de necrosis tumoral- $\alpha$ (TACE). ${ }^{15-17}$ Después se determinó que TACE se comportaba como un miembro de la familia de las ADAM, por lo que se le renombró como ADAM17. ${ }^{18,19}$ Se ha descrito que la ADAM17 es producida por varios tipos de células, incluyendo los linfocitos Ty monocitos. ${ }^{15,20}$

Por la escisión de citocinas de la superficie celular, ADAM17 regula su liberación dentro del microambiente local, contribuyendo al establecimiento de la inflamación. La ADAM17 es capaz de escindir ligandos y receptores altamente asociados con la inflamación, como el factor de necrosis tumoral- $\alpha$ y su receptor p55, el factor de crecimiento transformante- $\beta$, el receptor a interleucina- $1{ }^{17}$ y el ligando del receptor activador de NF- $\kappa B$ (RANKL). ${ }^{20,21} \mathrm{El}$ RANKL, un miembro de la familia del TNF- $\alpha$, se requiere para disparar la osteoclastogénesis y activar la reabsorción ósea. ${ }^{22}$ Hasta el momento se conoce que ADAM17 actúa sobre más de 80 sustratos en el rango de las citocinas, factores de crecimiento y moléculas de adhesión. ${ }^{19,23}$

Cerca de $10 \%$ de las proteínas de superficie celular se considera que pueden ser proteolíticamente cortadas, generando proteínas solubles. En relación a este mecanismo ADAM17 fue la primera proteasa caracterizada y se demostró que está formada por una secuencia con el extremo $\mathrm{N}$-terminal, seguida de un pro-dominio, una metaloproteinasa o dominio catalítico, un dominio de desintegrina, un dominio proximal membranal rico en cisteína, un solo dominio transmembranal y una porción citoplásmica (Figura 2). ${ }^{15,16}$

Es importante señalar que el pro-dominio $\mathrm{N}$-terminal de ADAM17 actúa como una chaperona y se piensa que inhibe la actividad catalítica de la enzima. El pro-dominio es removido por un corte secuencial por la proteína furina convertasa en dos sitios. ${ }^{24}$ El dominio proximal membranal es seguido por una secuencia corta, la cual está altamente conservada en el reino animal y esta secuencia es llamada dominio CANDIS (del inglés conserved ADAM seventeen dynamic interaction sequence). ${ }^{25} \mathrm{Se}$ ha demostrado que el dominio proximal está sujeto a un control regulador. Esta determinación estructural es capaz de proveer una isomerización de disulfuro, resultando en una conformación de abierta a cerrada. ${ }^{26}$ Esto restringe la interacción con sustratos y la accesibilidad de la fosfatidilserina, la cual actúa como un activador de ADAM17 (Figura 3). ${ }^{27}$

Debido a la gran cantidad de sustratos que tiene ADAM17, entre los cuales se incluye a las citocinas y factores de crecimiento, receptores barredores, moléculas de adhesión y otras proteínas transmembranales, se explica el involucramiento que éstas tienen en varios procesos patológicos. ${ }^{23}$

Estudios clínicos de la enfermedad periodontal claramente indican que existen niveles elevados de la expresión de RANKL en tejido periodontal. ${ }^{28-31}$ Las observaciones de que los niveles de RANKL están elevados 
en el líquido crevicular gingival (LCG) de pacientes con periodontitis y que ADAM17 puede escindir el RANKL unido a la membrana de una forma más eficiente que otras enzimas, ${ }^{20}$ sugiere la posibilidad de que ADAM 17 puede estar elevada en el LCG en la periodontitis. Dicho así, varios autores hipotetizaron que los niveles de ADAM17 estarían elevados en el LCG y en el tejido gingival de pacientes con periodontitis destructiva.

Por ejemplo, Bostanci y su equipo determinaron mediante el método de ELISA los niveles de ADAM17 en LCG de pacientes con periodontitis y gingivitis y encontraron una alta concentración de ADAM17 en los pacientes con periodontitis crónica (PC) y periodontitis agresiva (PA) en comparación con el grupo de gingivitis. De la misma forma, los niveles de ADAM17 exhibieron una correlación positiva con la profundidad al sondeo, niveles de inserción clínica y con el RANKL en el LCG, por lo que concluyeron que la correlación positiva entre los niveles de RANKL y ADAM17 en pacientes con periodontitis indican una asociación de esta enzima con la pérdida de hueso alveolar. ${ }^{32}$

Por su parte, Lee y sus colegas propusieron investigar si ADAM17 podía servir o no como un biomarcador en diferentes estados de la periodontitis y determinar el efecto de ADAM17 sobre la expresión de RANKL y osteoprotegerina (OPG) en células osteoblásticas. Por lo que analizaron los niveles de ADAM17 en el LCG y tejido gingival proveniente de los pacientes con periodontitis y confirmaron que los niveles de ADAM17 se encontraron más elevados en los pacientes con periodontitis moderada en comparación con los sujetos sanos en ambos tipos de muestras mediante la técnica de ELISA. Por otra parte, para entender los efectos de ADAM17 sobre la expresión del RANKL soluble (RANKLs) y OPG en osteoblastos, estos investigadores trataron la línea celular MG63 con ADAM17 recombinante en cultivos celulares y después cuantificaron el RANKLs y el OPG mediante la técnica de Western blot, encontrando un aumento del RANKLs y una disminución del OPG. Estos datos sugieren que ADAM17 puede inducir la expresión de RANKL y promover la osteoclastogénesis, dicho así, influir en la progresión y el desenlace de la periodontitis. ${ }^{33}$

Del mismo modo, Kinoshita y su grupo estudiaron el RNA mensajero (RNAm) de la ADAM17 en muestras de epitelio de la mucosa oral de pacientes con gingivitis, periodontitis temprana, moderada y avanzada, así como en sujetos sanos por RT-PCR en tiempo real, y encontraron un aumento del RNAm de ADAM17 en los pacientes con periodontitis moderada y avanzada en comparación con los sujetos sanos y pacientes con gingivitis. Del mismo modo, encontraron una correlación significativamente positiva de la ADAM17 con la profundidad al sondeo. Estos investigadores concluyen que la alta expresión del RNAm de la ADAM17 en el epitelio de la mucosa oral de pacientes con periodontitis avanzada puede estar relacionada con el papel de la ADAM17 en el señalamiento del receptor del factor de crecimiento epidérmico (EGFR), el cual participa en mantener la homeostasis de las barreras

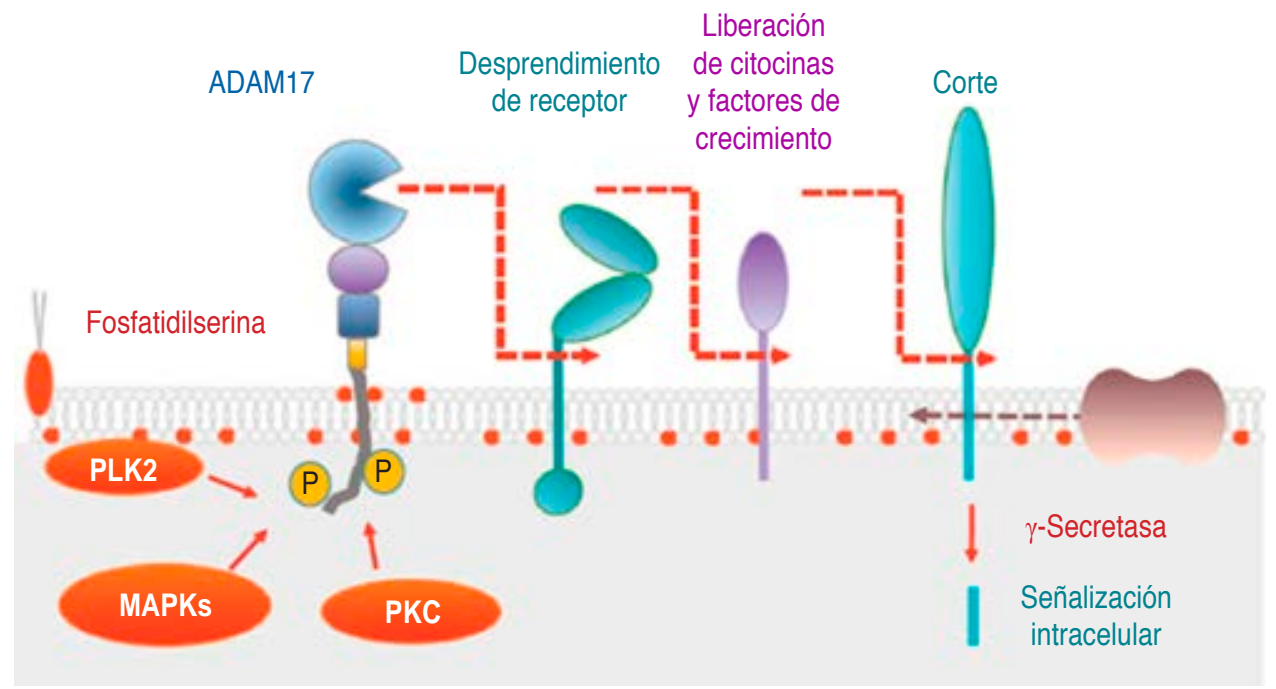

Figura 3: Esquema general de la actividad de escisión de ADAM17. La función de ADAM17 está regulada por la fosforilación del tallo citoplasmático por las cinasas intracelulares como PLK2, MAPK, PKC. Se requiere de la transferencia de la fosfatidilserina a la membrana para la activación de la ADAM17. Las actividades de la ADAM17 incluyen la escisión de receptores, citocinas y factores de crecimiento, llevando a un rasgamiento de las proteínas de membrana por la enzima $\gamma$-secretasa, la cual corta en un solo paso a las proteínas de membrana sin el dominio transmembranal.

Modificada de: Zunke F et al. $2017 .{ }^{19} \mathrm{ADAM}$ = del inglés a disintegrin and metalloproteinase; PLK2 = serina/treonina proterina cinasa 2; MAPK = croteína quinasa activada por mitógenos; $\mathrm{PKC}=$ proteína cinasa $\mathrm{C}$. 
epidérmicas de la mucosa oral y de esta manera aumentar los mediadores de la inflamación para promover el deterioro de la enfermedad periodontal. ${ }^{34}$

Por otra parte, se conoce que la IL-23 es una citocina proinflamatoria que está compuesta por la subunidad IL-12p40 y la p19. ${ }^{35}$ La IL-23 es un factor clave para el desarrollo de las células Th17 $7^{36}$ y controla respuestas antimicrobianas y antifúngicas, pero está críticamente involucrado en la patogénesis de los desórdenes inflamatorios. ${ }^{37}$ El complejo receptor a IL-23 (IL-23R) está compuesto por una subunidad llamada IL-23R y por la cadena B1 común con el receptor a IL-12 (IL-12Rß1). ${ }^{38,39}$ El corte y empalme alternativo genera una forma soluble antagonista del IL-23R (IL-23Rs). Sin embargo, existe otro mecanismo para formar IL-23Rs descrito por Franke y Colaboradores, quienes publicaron que la ADAM17 puede escindir al IL-23R a través de la escisión del ectodominio unido a la membrana. ${ }^{40}$ En este sentido se determinó que existe un aumento significativo en los niveles de IL$23 R s$ en plasma de pacientes con periodontitis agresiva y periodontitis crónica en comparación con sujetos sanos. Estos resultados sugieren que probablemente la ADAM17 esté escindiendo al IL-23R, convirtiéndolo en la forma soluble que se ha detectado en los pacientes con periodontitis, ${ }^{41}$ debido a que ya se ha demostrado un aumento de la ADAM17 en el LCG y en el tejido gingival de los pacientes con periodontitis. ${ }^{32,34}$

Es importante señalar que además se ha confirmado un aumento de la expresión de ADAM17 a nivel de proteína en muestras de tejido gingival de pacientes con periodontitis en comparación de sujetos sanos a través de la técnica de Western blot. ${ }^{42}$

\section{CONCLUSIÓN}

El aumento de ADAM17 en el LCG, así como la elevada expresión del RNAm y la proteína de ADAM17 en el tejido gingival de pacientes con periodontitis, demuestra que esta molécula está involucrada en el proceso de la osteoclastogénesis, debido a que se ha demostrado que actúa sobre el aumento de la expresión del RANKL, IL$23 R$ y del receptor a TNF- $\alpha$.

\section{REFERENCIAS}

1. Hajishengallis G. Periodontitis: from microbial immune subversion to systemic inflammation. Nat Rev Immunol. 2015; 15 (1): 30-44. doi: 10.1038/nri3785.

2. Kinane DF, Stathopoulou PG, Papapanou PN. Periodontal diseases. Nat Rev Dis Primers. 2017; 22 (3): 17038. doi: 10.1038/ nrdp.2017.38.

3. Wolsberg TG, Primakoff P, Myles DG, White JM. ADAM, a novel family of membrane proteins containing a disintegrin and me- talloproteinase domain: multipotential functions in cell-cell and cell-matrix interactions. J Cell Biol. 1995; 131: 275-278.

4. Edwards DR, Handsley MM, Pennington CJ. The ADAM metaIloproteinases. Mol Aspects Med. 2008; 29 (5): 258-289. doi: 10.1016/j.mam.2008.08.001.

5. Endres K, Fahrenholz F. Regulation of $\alpha$-secretase ADAM10 expression and activity. Exp Brain Res. 2012; 217: 343-352.

6. Reiss K, Saftig P. The "a disintegrin and metalloprotease" (ADAM) family of sheddases: physiological and cellular functions. Semin Cell Dev Biol. 2009; 20: 126-137.

7. Hynes RO, Lively JC, McCarty JH, Taverna D, Francis SE, HodivalaDilke $\mathrm{K}$ et al. The diverse roles of integrins and their ligands in angiogenesis. Cold Spring Harb Symp Quant Biol. 2002; 67: 143-153.

8. Saftig P, Reiss K. The "a disintegrin and metalloproteases" ADAM10 and ADAM17: novel drug targets with therapeutic potential? Eur J Cell Biol. 2011; 90 (6-7): 527-535. doi: 10.1016/j.ejcb.2010.11.005.

9. Smith KM, Gaultier A, Cousin H, Alfandari D, White JM, DeSimone DW. The cysteine-rich domain regulates ADAM protease function in vivo. J Cell Biol. 2002; 159: 893-902.

10. Janes PW, Saha N, Barton WA, Kolev MV, Wimmer-Kleikamp SH, Nievergall E et al. ADAM meets Eph: an ADAM substrate recognition module acts as a molecular switch for ephrin cleavage in trans. Cell. 2005; 123: 291-304.

11. Seals DF, Courtneidge SA. The ADAM family of metalloproteases: multidomain proteins with multiple functions. Genes Dev. 2003; 17: 7-30.

12. Hartmann D, de Strooper B, Serneels L. Cloning of a disintegrin metaloproteinase that processes precursor tumour-necrosis factor-alpha. Nature. 1997; 385: 733-736.

13. Qi H, Rand MD, Wu X, Sestan N, Wang W, Rakic P et al. Processing of the notch ligand delta by the metalloprotease Kuzbanian. Science. 1999; 283: 91-94.

14. Rocks N, Paulissen G, El Hour M, Quesada F, Crahay C, Gueders $M$ et al. Emerging roles of ADAM and ADAMTS metalloproteinases in cancer. Biochimie. 2008; 90: 369-379.

15. Black RA, Rauch CT, Kozlosky CJ, Peschon JJ, Slack JL, Wolfson MF et al. A metalloproteinase disintegrin that releases tumournecrosis factor-alpha from cells. Nature. 1997; 20385 (6618): 729-733. doi: 10.1038/385729a0.

16. Moss ML, Jin SL, Milla ME, Bickett DM, Burkhart W, Carter HL et al. Cloning of a disintegrin metalloproteinase that processes precursor tumour-necrosis factor-alpha. Nature. 1997; 385 (6618): 733-736. doi: 10.1038/385733a0.

17. Reddy P, Slack JL, Davis R, Cerretti DP, Kozlosky CJ, Blanton RA et al. Functional analysis of the domain structure of tumor necrosis factor-alpha converting enzyme. J Biol Chem. 2000; 275 (19): 14608-14614. doi: 10.1074/jbc.275.19.14608.

18. Müllberg J, Althoff K, Jostock T, Rose-John S. The importance of shedding of membrane proteins for cytokine biology. Eur Cytokine Netw. 2000; 11 (1): 27-38.

19. Zunke F, Rose-John S. The shedding protease ADAM17: physiology and pathophysiology. Biochim Biophys Acta Mol Cell Res. 2017; 1864 (11 Pt B): 2059-2070. doi: 10.1016/j.bbamcr.2017.07.001.

20. Lum L, Wong BR, Josien R, Becherer JD, Erdjument-Bromage $H$, Schlondorff $J$ et al. Evidence for a role of a tumor necrosis factor-alpha (TNF-alpha)-converting enzyme-like protease in shedding of TRANCE, a TNF family member involved in osteoclastogenesis and dendritic cell survival. J Biol Chem. 1999; 274 (19): 13613-13618. doi: 10.1074/ jbc.274.19.13613.

21. Nakashima T, Kobayashi Y, Yamasaki S, Kawakami A, Eguchi K, Sasaki $\mathrm{H}$ et al. Protein expression and functional difference of membrane-bound and soluble receptor activator of NF-kappa B 
ligand: modulation of the expression by osteotropic factors and cytokines. Biochem Biophys Res Commun. 2000; 275 (3): 768775. doi: 10.1006/bbrc.2000.3379.

22. Teitelbaum SL. Bone resorption by osteoclasts. Science. 2000; 289 (5484): 1504-1508. doi: 10.1126/science.289.5484.1504.

23. Scheller J, Chalaris A, Garbers C, Rose-John S. ADAM17: a molecular switch to control inflammation and tissue regeneration. Trends Immunol. 2011; 32 (8): 380-387. doi: 10.1016/j. it.2011.05.005.

24. Wong E, Cohen T, Romi E, Levin M, Peleg Y, Arad U et al. Harnessing the natural inhibitory domain to control TNF $\alpha$ Converting Enzyme (TACE) activity in vivo. Sci Rep. 2016; 6: 35598. doi: 10.1038/srep35598.

25. Düsterhoft S, Hobel K, Oldefest M, Lokau J, Waetzig GH, Chalaris A et al. A disintegrin and metalloprotease 17 dynamic interaction sequence, the sweet tooth for the human interleukin 6 receptor. J Biol Chem. 2014; 289 (23): 16336-16348. doi: 10.1074/jbc. M114.557322.

26. Düsterhoft S, Jung S, Hung CW, Tholey A, Sonnichsen FD, Grotzinger J et al. Membrane-proximal domain of a disintegrin and metalloprotease-17 represents the putative molecular switch of its shedding activity operated by protein-disulfide isomerase. J Am Chem Soc. 2013; 135 (15): 5776-5781. doi: 10.1021/ ja400340u.

27. Sommer A, Kordowski F, Büch J, Maretzky T, Evers A, Andra J et al. Phosphatidylserine exposure is required for ADAM17 sheddase function. Nat Commun. 2016; 7: 11523. doi: 10.1038/ ncomms11523.

28. Vernal R, Chaparro A, Graumann R, Puente J, Valenzuela MA, Gamonal J. Levels of cytokine receptor activator of nuclear factor kappa B ligand in gingival crevicular fluid in untreated chronic periodontitis patients. J Periodontol. 2004; 75 (12): 1586-1591. doi: 10.1902/jop.2004.75.12.1586.

29. Nishijima Y, Yamaguchi M, Kojima T, Aihara N, Nakajima R, Kasai K. Levels of RANKL and OPG in gingival crevicular fluid during orthodontic tooth movement and effect of compression force on releases from periodontal ligament cells in vitro. Orthod Craniofac Res. 2006; 9 (2): 63-70. doi: 10.1111/j.16016343.2006.00340.x

30. Bostanci N, Ilgenli T, Emingil G, Afacan B, Han B, Toz H et al. Gingival crevicular fluid levels of RANKL and OPG in periodontal diseases: implications of their relative ratio. J Clin Periodontol. 2007; 34 (5): 370-376. doi: 10.1111/j.1600051X.2007.01061.x.

31. Bostanci N, Ilgenli T, Emingil G, Afacan B, Han B, Toz H et al. Differential expression of receptor activator of nuclear factor-kappa B ligand and osteoprotegerin mRNA in periodontal diseases. J Periodontal Res. 2007; 42 (4): 287-293. doi: 10.1111/j.16000765.2006.00946.x.

32. Bostanci N, Emingil G, Afacan B, Han B, Ilgenli T, Atilla G et al. Tumor necrosis factor-alpha-converting enzyme (TACE) levels in periodontal diseases. J Dent Res. 2008; 87 (3): 273-277. doi: $10.1177 / 154405910808700311$.

33. Lee JH, Choi YJ, Heo SH, Lee JM, Cho JY. Tumor necrosis factor- $\alpha$ converting enzyme (TACE) increases RANKL expression in osteoblasts and serves as a potential biomarker of periodontitis. BMB Rep. 2011; 44 (7): 473-477. doi: 10.5483/BMBRep.2011.44.7.473.

34. Kinoshita N, Awano S, Yoshida A, Soh I, Ansai T. Periodontal disease and gene-expression levels of metalloendopeptidases in human buccal mucosal epithelium. J Periodontal Res. 2013; 48 (5): 606-614. doi: 10.1111/jre.12045.

35. Floss DM, Moll JM, Scheller J. IL-12 and IL-23-close relatives with structural homologies but distinct immunological functions. Cells. 2020; 9 (10): 2184. doi: 10.3390/cells9102184.

36. Ouyang W, Kolls JK, Zheng Y. The biological functions of T helper 17 cell effector cytokines in inflammation. Immunity. 2008; 28 (4): 454-467. doi: 10.1016/j.immuni.2008.03.004.

37. Cosmi L, Maggi L, Santarlasci V, Liotta F, Annunziato F. T helper cells plasticity in inflammation. Cytometry A. 2014; 85 (1): 36-42. doi: 10.1002/cyto.a.22348.

38. Presky DH, Yang H, Minetti LJ, Chua AO, Nabavi N, Wu CY et al. A functional interleukin 12 receptor complex is composed of two beta-type cytokine receptor subunits. Proc Natl Acad Sci U S A. 1996; 93 (24): 14002-14007. doi: 10.1073/pnas.93.24.14002.

39. Parham C, Chirica M, Timans J, Vaisberg E, Travis M, Cheung J et al. A receptor for the heterodimeric cytokine IL-23 is composed of IL12Rbeta1 and a novel cytokine receptor subunit, IL-23R. I Immunol. 2002; 168 (11): 5699-5708. doi: 10.4049/jimmunol.168.11.5699.

40. Franke M, Schroder J, Monhasery N, Ackfeld T, Hummel TM, Rabe $\mathrm{B}$ et al. Human and murine interleukin 23 receptors are novel substrates for a disintegrin and metalloproteases ADAM10 and ADAM17. J Biol Chem. 2016; 91 (20): 10551-10561. doi: 10.1074/jbc.M115.710541.

41. Rivadeneyra-Burgos C, Rodríguez-Montaño R, Ruíz-Gutiérrez AC, Martínez-Rodríguez VC, Meléndez-Ruiz JL, Pita-López ML et al. Determination of levels of IL-23 soluble receptor in serum and plasma of patients with chronic and aggressive periodontitis. Rev Mex Periodontol. 2017; 8 (1): 5-10.

42. Guardiola CJA, Clemente-Napimoga JT, Martinez EF, Abdalla HB, Peruzzo DC, Joly JC et al. DC-STAMP and TACE levels are higher in patients with periodontitis. Braz Dent J. 2020; 31 (2): 122-126. doi: 10.1590/0103-6440202002939.

\section{Correspondencia: \\ Celia Guerrero Velázquez \\ Sierra Mojada Núm. 950, Col. Independencia, 44340, Guadalajara, Jalisco, México. \\ Teléfono: 33 1052-5800, Ext. 33748 y 33749 \\ E-mail: celiagv2001@yahoo.com.mx}

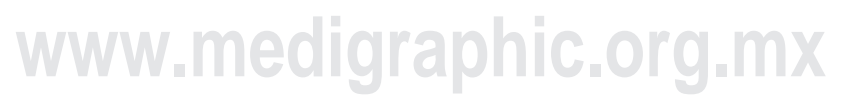

